\title{
REVIEW
}

\section{The Human Rights Act 1998 and medical treatment: time for re-examination}

\section{E Hagger}

\section{Arch Dis Child 2004;89:460-463. doi: 10.1136/adc.2003.037218}

There was much speculation about the potential impact of the Human Rights Act 1998 on established principles of health care practice. Now the statute has been "bedded in $^{\prime \prime}$, predictions that existing good practice would not fall foul of the legislation provided it was demonstrable, have proved to be broadly accurate. This paper illustrates the approach of the courts to areas of difficulty related to medical treatment and highlights, in particular, possible challenges under the Act by the mature minor whose refusal of treatment has been overridden. This includes a discussion of those with learning disabilities, and reference is also made to the implications of the legislation in relation to confidentiality and child protection.

Correspondence to:

Ms L E Hagger,

Department of Law, Crookesmoor Building, University of Sheffield, Conduit Road, Sheffield S10 1FL, UK;

L.E.Hagger@shef.ac.uk

Accepted

3 September 2003
$\mathrm{T}$ he Human Rights Act 1998 (HRA) incorporates much of the European Convention on Human Rights (ECHR) into domestic law. Received wisdom suggested that, in the light of earlier Strasbourg case law, ${ }^{1}$ existing good health care practice would not fall foul of the legislation but would need to be demonstrated explicitly. The mental health arena has seen a number of HRA challenges, not least because procedural safeguards must be in place. Case law concerning resource allocation decisions by local authorities suggests that the courts continue to be sympathetic to the difficulties in making such decisions and will look favourably on public bodies who consider the human rights implications explicitly and seek to minimise the effects on individuals. ${ }^{2}$ The law will continue to provide a framework within which to work but will still leave much to clinical judgement. Seeking advice from solicitors is not always helpful in difficult cases because their opinions may be conflicting ${ }^{3}$ and/ or over-cautious. ${ }^{4}$ Health professionals are well equipped themselves to make ethically sound decisions that meet legal requirements with appropriate training and support. How will the HRA affect medical decision making in relation to children?

\section{MEDICAL TREATMENT AND DISPUTES}

The UK courts have generally supported reasoned, medical decision making ${ }^{5}$ and this looks set to continue under the auspices of the HRA. In NHS Trust $v$ D \& Others, ${ }^{6}$ parents challenged a decision by the medical team that it was in the best interests of a severely ill and disabled child not to ventilate him but to offer palliative care. The court supported the professional view that the minimal quality of life he would enjoy for only a short time was outweighed by the pain and suffering that would be caused by the intervention. This meant that the right to life enshrined in Article 2 of the ECHR would not be breached and that the right to die with dignity arising from Article 3 of the ECHR, ${ }^{7}$ which includes the right not to be subjected to inhumane and degrading treatment, would be protected. This approach, looking at the overall best interests of the child, will also be the guiding principle for the court where the dispute is between the parents themselves. ${ }^{8}$

Where objections to medical treatment are based on religious convictions, parents may attempt to rely on the right to private and family life under Article 8 and the right to manifest religion under Article 9 of the ECHR. However, neither of these are absolute rights and, given the commitment to the sanctity of life and best interest considerations exhibited in earlier cases, the ability to protect the rights and freedoms of others, here the child, could be relied on to validate medical decisions.

That being said, it is important to note that national courts have emphasised the importance of involving parents in decision-making. $R v$ Portsmouth Hospitals NHS Trust ex parte Glass ${ }^{10}$ demonstrates how the ideal is for doctors and parents to agree in a climate of full information, consultation, negotiation and compromise but, where this is no longer possible, the court should adjudicate. Parental suggestions may be overruled if it does not concur with medical opinion. ${ }^{11}$ Strasbourg case law suggests that the HRA does provide a much stronger means whereby families may challenge any perceived lack of involvement in the decision making process. ${ }^{12}$ Those close to the patient may be a very useful means to ascertain an incompetent patient's preferences. Where the Gillick $^{13}$ or otherwise competent child is concerned, such involvement must be with the child's approval. Effective and candid communication prevents unnecessary delay, is likely to be ethical and in the child's best interests. Communication, including counselling, skills can be as important as clinical expertise. Counselling facilitates the child and/or the parents/carers' ability to make reasoned choices and accommodate to new circumstances. ${ }^{14}$

How may the HRA impact on other key areas of concern in paediatric practice? The NHS Litigation Authority believes a challenge to parental consent to treatment where this overrides a competent child's refusal is probable. ${ }^{15}$ 


\section{CONSENT AND THE MATURE MINOR}

The adult, competent, and conscious patient must consent to any bodily contact, otherwise the health professional may be vulnerable to a legal action in the tort of battery whether or not the individual has suffered harm. ${ }^{16}$ The law's response to calls for a similar acknowledgement in relation to children has been more muted. Those with parental responsibility and the courts may consent to treatment for children. A child who is deemed "Gillick competent", that is, of sufficient maturity and understanding, may consent to treatment but $R e R^{17}$ made it clear that they do not have the corollary right to refuse. Sixteen and seventeen year old children may consent to treatment under section 8 of the Family Law Reform Act 1969 but, as illustrated by $R e W,{ }^{18}$ also cannot refuse.

It is the coherence of these pre-HRA cases relating to consent and the mature minor that has been questioned, less for the outcomes, which are rather more difficult to dispute, than for the reasoning which undermines children's autonomy rights. ${ }^{19}$ Re $R$ has been criticised for its lack of articulation as to why minority of itself justifies overriding a judgement of a Gillick competent child. In both Re $R$ and Re $W$ the adolescents' conditions were not unique to age because they would have failed the adult mental capacity test established in $\operatorname{Re} C{ }^{20}$ thus leading to an inability to make a proper choice of treatment.

In these cases, age allowed the courts to avoid the use of mental health legislation to prevent stigmatisation. Arguably, in so doing, they perpetuated stigmatisation and undermined respect for the autonomy of children. Closer examination of $R e W$ does reveal a commitment to considering the minor's preferences. Attempts have been made to assess the idea of fully autonomous choice in $\operatorname{Re} T,{ }^{21}$ but this is an aspiration and, in reality, only maximally autonomous choice is possible. A more constructive approach would include the notion that adolescent welfare does not have to be sacrificed if society accepts the notion of evolving autonomy which makes far more sense than imposing arbitrary age limits with respect to capacity. ${ }^{22}$ The approach adopted in $\operatorname{Re} T$, where there is a presumption of capacity allowing for the right to self-determination even if this conflicts with society's interest in preserving life, is suggested as equally relevant for the maturing minor. The fundamental factor in autonomous decision making, the ability to make a choice, would extend the notion of Gillick competence by taking into account more than the ability to understand.

\section{The need to take children's autonomy interests seriously}

The right to private life under Article 8 ECHR protects autonomy interests and would be at the centre of any claim now made by mature minors who believe their refusal of treatment should not be overridden. ${ }^{23}$ A more sophisticated assessment of competence may assist in acknowledging these autonomy interests.

An important consequence of a strong legal commitment to privacy is that it serves as a reminder of its significance and will be translated in other contexts. This has a particular resonance in the health care setting given the number of potential invasions of privacy. Anyone who doubts its importance to adolescents and younger children, merely needs to conduct a survey within the hospital setting. Robust legal statements about privacy seems ever more possible given common law developments in the light of the HRA in that there has been an explicit recognition of the right to privacy in English law for the first time, ${ }^{24}$ albeit attached to the law of confidence. Quite what the parameters of this might be will need further judicial exploration, but there is a need to extend this area so that the right to privacy becomes a "stand alone" right since it encompasses broader notions of autonomy interests.

The United Nations Convention on the Rights of the Child 1989 (UNCRC), ratified by the UK, underpins the legal framework and is a clear indication that today's society recognises the need to consult children and respond to their views. However, it is arguable that some of the key Articles of the UNCRC are not fully reflected in practice which can be inconsistent and incoherent. In particular, Article 12:

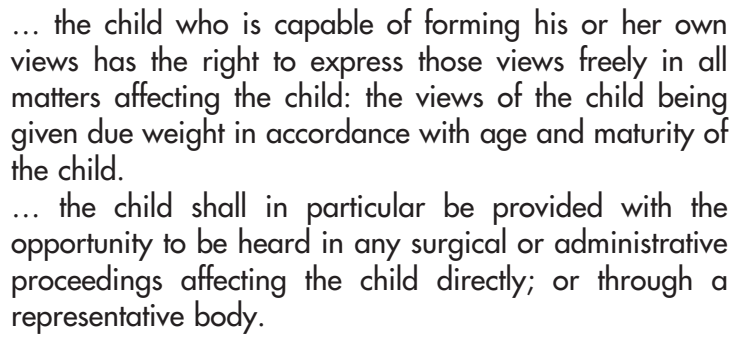

This does not necessarily reflect a lack of commitment to children's rights but a lack of confidence in assessing children's competence in relation to decisions with very significant implications for that child. Such trepidation is entirely understandable, not least because of the litigious climate of current practice. However, the present focus on quality issues in the report arising from the inquiry into children's heart surgery at Bristol Royal Infirmary, ${ }^{25}$ which noted the lack of priority given to children's issues, and the HRA which gives rights to children, means that this area must be revisited. With this in mind, and with the introduction of a National Service Framework for children to establish clear standards for services, perhaps the ethical dimension of working with children will receive long overdue attention.

There are many calls for increased levels of information to be provided for children and their families, including the Department of Health (DoH) consent forms and accompanying documentation, ${ }^{26}$ but this is a meaningless exercise for older children if they are not then given adequate opportunities to exercise appropriate choices. There have been excellent documents provided by professional bodies reminding of the need to facilitate the decision making process in this way. ${ }^{27}$ The DoH consent forms set out the law in relation to children in an accessible way and provide an excellent format for determining competence, alerting the assessor not to make assumptions based on age or apparent ability alone. Demands on time and resources will not allow the luxury of detailed psychological assessment in all cases and, indeed, this would not be appropriate where the implications of treatment are less significant. It is the practical guidance that health professionals need, particularly during the early part of their careers. How may the HRA support the reflective health care professional, committed to an ethical approach?

\section{The competency issues for health professionals}

The law necessarily focuses on the final stage of decision making where there has been a refusal of medical intervention. Health professionals need to be aware of the legal framework within which they must operate but should not ignore the process that precedes judicial decisions. Details of relevant cases could help because these highlight the need for, inter alia, involving children and giving credence to their views. ${ }^{28}$ Professionals need to be trained in child development so that they understand how children think and understand. Involving the child is a transactional process that will alter the perspective of the adult and could affect their decision making significantly. ${ }^{29}$ The process itself can empower 
children and their carers as they are helped to face difficult decisions, adding to their confidence and competence, as well as providing a thorough means of assessing children's capacity. This should include taking account of the children's experience of their illness and how this might have had an impact on their maturity and understanding. ${ }^{30}$ Using age and traditional measures of general intelligence alone is a poor measure of a child's capacity to have sufficient understanding and de-emphasises the importance of the social component of cognitive development and, thereby, the impact of contextual factors. ${ }^{31}$

It should also be noted that although one's privacy interests are necessarily circumscribed when entering the public domain, they do not have to be lost in their entirety. All efforts should be made to preserve these interests wherever possible, engaging the individual at every opportunity. There are some examples of excellent practice where the child is given every opportunity to decide their optimum treatment, at all stages, decisions big and small, with all the disciplines involved in their care. ${ }^{32}$ Given the frequency with which competency issues arise in the medical context, protocols for assessing capacity would be advisable. These could include wide ranging factors to be taken into account and could form part of an integrated care pathway where a multidisciplinary approach is adopted in assessing, planning, implementing, monitoring, and evaluating care in collaboration with the patient and family. The use of a Clinical Ethics Committee in developing policies or dealing with individual cases is another way of developing an enhanced approach to the medical treatment of children. What better way is there of demonstrating ethical decision making fully in compliance with the HRA?

Where there is no mental disability present, will society countenance decisions being made by adolescents that may be seen as defeating their self-interest? Legal advice, in practice, is likely to advocate using mental health legislation to treat psychiatric and physical conditions where the latter can be said to be ancillary to the mental disturbance or to seek a court declaration as to capacity where that may be seen as finely balanced. ${ }^{33}$ Notwithstanding the real stigmatisation experienced by those subject to "sectioning", the mental health legislation does, at least, provide procedural safeguards for the individual.

Treatment rejection at some point in a chronic illness by adolescents followed by acceptance is a well observed phenomenon. Nevertheless, the proposition that the mature minor cannot refuse life saving treatment could be harder to sustain in cases where there may be limited success and/or the treatment itself is very distressing. Certainly in those cases where the condition is not life threatening but parents want their children to undergo painful treatment, even relatively young children should be fully engaged in the decision making process to ensure full commitment. Forcible treatment which may involve restraint or even detainment needs strong justification and consistency of approach. The reality for practitioners is that without the cooperation of the mature minor, many procedures will be impossible to administer, thus increased communication with young patients is essential. Confidence in dealing with these difficult situations would be greatly enhanced if appropriate, multidisciplinary training is provided to address effective, lawful methods of restraint within the context of a case study approach so that flexible policies may be developed to take account of the age, size, and condition of the child. Mental health services can often offer such support.

\section{Dealing with learning disabilities}

Excellent communication skills are vital when working with the learning disabled who will need additional explanation and reassurance. A broad based approach, engaging with all those close to the patient subject to the latter's approval, will be particularly helpful here to ensure a thorough understanding of the patient. The DoH consent guidance in this area provides a useful synthesis of good practice and relevant law. Assumptions must not be made that having learning difficulties necessarily means that patients can make no decisions for themselves. Straightforward procedures might be within their capability to understand with appropriate accessible information and support. They can then decide whether they wish to proceed. In the case of more sophisticated interventions, it may become necessary to act in the patients' best interests. This should be determined from the patient's perspective, perhaps using the views of those close to the patient. The earlier point about forcible treatment has an equal bearing here. Significant attempts should be made to persuade the patient but, where this becomes impossible, only the most compelling reasons would justify any kind of restraint. Life saving procedures and those having a very significant impact on the quality of the patient's life would fall into this category.

\section{CONFIDENTIALITY AND CHILD PROTECTION}

The Article 8 (ECHR) right to private life includes a provision for the consideration of the needs of others. As a result, confidentiality is an area that may require an approach that incorporates such a notion in a wider sense than has previously been the case. Here, the focus will be on confidentiality in relation to child protection.

A key recommendation of the Laming Report into Victoria Climbié's death was that the law relating to the sharing of information among professional groups should be clarified for those involved in child protection. ${ }^{34}$ While this would be helpful, existing guidance offers accessible information setting out the requirements of the relevant law including the common law, the Children Act 1989, the HRA, and the Data Protection Act 1998 (DPA).$^{35}$ What might be particularly useful would be to provide a synthesis of these areas highlighting particular issues for professionals in the field. There will still, necessarily, be much left to the individual professional to decide when legal requirements may be overridden. Experience and developing expertise provides the confidence needed to offer robust justification for such courses of action. Multidisciplinary training and support will enhance this process, not least because the best decisions are likely to be made on a team basis.

It is clear that there is an overriding statutory duty under the Children Act 1989 and a common law duty to safeguard children by sharing information with relevant agencies where there is manifest evidence of child abuse. ${ }^{36}$ This should be subject to appropriate protocols to safeguard relevant interests $^{37}$ and to ensure that all parties are as fully engaged in the process as possible. Section 115 of the Crime and Disorder Act 1998 and an exemption in the DPA in section 29 allows the legitimate disclosure of information in the interests of criminal justice, notwithstanding the overarching requirement of obtaining consent for disclosure under the DPA. To be legitimate, rigorous public interest arguments need to be forthcoming. ${ }^{38}$

The common law also provides for the public interest exception where there is a risk of death or serious harm. ${ }^{39}$ Again, this allows for considerable subjective interpretation. Professional guidelines may be helpful in this regard, particularly in relation to addressing the medical needs of patients, while still leaving much to the judgement of the individual professional. ${ }^{40}$ It should be noted that Gillick competent children are entitled to the same duty of confidence as adults, unless it is believed that a child seeking advice is being exploited or abused. 
The right to private life under Article 8 of the ECHR will also be engaged where there is a disclosure of personal information. Disclosure will be justifiable where it is occurring to safeguard children because of the provisions in Article 8(2) for the protection of health, morals, the rights and freedoms of others, and for the prevention of disorder or crime. In addition, information must be adequate, relevant, not excessive in relation to the purpose for which it is held, and must not be held longer than is necessary for that purpose to satisfy both the DPA and the HRA.

A more difficult area is where health professionals have their suspicions raised but need to undertake further investigation to justify such disclosure. The obtaining of consent to do this, and to disclose personal information to other agencies, will satisfy all legal requirements. Many clinicians are very skilled in obtaining the requisite consents notwithstanding the potentially fraught nature of the exercise. If successful in obtaining consent, it is self evident that thorough records should be kept of the entire process. Where obtaining consent is not possible, the right to confidentiality may be breached under all the legal provisions broadly on the basis of the wider public interest. It is the interpretation of this which can cause difficulty not least because of disagreement as to the level of risk needed to justify this step. This suggests that a detailed discussion needs to take place so that appropriate risk assessment can be demonstrated in the event of a later legal challenge. It is highly probable that the courts would support an approach that illustrates reasonable endeavours on the part of relevant professionals.

\section{CONCLUSIONS}

We have seen that the law provides a framework within which to work but necessarily leaves much to clinical judgement and that health professionals, familiar with all aspects of the case, are more than capable of making ethically sound decisions that meet legal requirements. The potential for possible legal challenges and the demands of the clinical governance agenda must mean that both clinical and ethical approaches to health care are examined at both a local and national level. There are a number of ways of achieving legal and ethical decision making. The ultimate decision about a patient's treatment remains with the clinician in charge. Nevertheless, the more other members of the health care team, the patient and those close to the patient, where appropriate, are involved in informing that decision, it is self evident that the quality of the decision will be enhanced. This, in turn, will be informed by relevant guidelines, policies, and protocols which should be periodically revisited to ensure current practice conforms to these at minimum and, further, that these approaches meet the needs of the local setting. This approach is relevant for assessing competence and determining the best interests of the patient and others.

\section{REFERENCES AND NOTES}

1 To be taken into account under section 2, HRA.

$2 \mathrm{R}$ (on the application of Dudley and others) v. East Sussex County Council [2003] EWHC 803 Admin., 16 April 2003.

3 AK (Adult Patient) (Medical Treatment: Consent) [2001] 1 FLR 129.

4 Slowther A, Bunch C, Woolnough B, et al. Ethox (Oxford Centre for Ethics and Communication in Health Care Practice), University of Oxford, The Nuffield
Trust, "Clinical ethics support in the UK: a review of the current position and likely development". Clinical Ethics Committees Supplement. Journal of Medical Ethics 2001;27(suppl 1).

$5 \operatorname{Re} C$ (a minor) (medical treatment [1998] 1 FLR 384.

6 [2000] 2 FLR 677.

7 Following D v. United Kingdom [1997] 24 EHRR 423.

$8 \mathrm{ReJ}$ (Specific Issues Orders: Muslim Upbringing and Circumcision) [1999] 2 FLR 678.

9 See, for example, Re R (A Minor) (Blood Transfusion) [1993] 2 FLR 757.

10 [1999] 2 FLR 905; approach affirmed by the European Court of Human Rights, which decided a hospital's failure to involve the courts in the dispute between a mother and the doctors caring for her son constituted a breach of Article 8 ECHR: Glass V. UK, Application No. 61827/00, 10 March 2004.

11 Re MM (Medical Treatment) [2000] 1 FLR 224 where Russian parents wanted the UK doctors caring for their immunodeficient child to adopt the course of treatment advocated in their home country.

12 W v UK (1987) 10 EHRR 29 and TP \& KM v United Kingdom EHHR [2001] 2 FLR 549.

13 Gillick v West Norfolk and Wisbech Area Health Authority [1985] 3 All ER 402, HL.

14 Palmer Barnes F, Murdin L, eds. Values and ethics in the practice of psychotherapy and counselling. Buckingham: Open University Press, 2001

15 www.nhsla.com: Human Rights Act Information Service.

16 www.nhsla.com: Human Rights

17 See, for example, Re R (A Minor) (Wardship: Consent to Medical Treatment) [1991] 4 All ER 177, CA.

18 Re W (A Minor) (Wardship: Consent to Medical Treatment) [1992] 4 All ER 627, CA.

19 Brazier M, Bridge C. Coercion or caring: analysing adolescent autonomy. 16 Legal Studies 84, 1996.

$20 \operatorname{Re} C$ (Adult: Refusal of Medical Treatment [1994] 1 All ER 819 in which Thorpe $\mathrm{J}$ set out the questions to be asked in determining mental competence: could the patient comprehend and retain the treatment information, did the patient believe the information, and could he weigh it in the balance to arrive at a choice? M Brazier and C Bridge.

$21 \operatorname{Re} T$ [1992] supra at n. 14.

22 Brazier M, Bridge C. supra n. 18 discussing J. Harris. The value of life. Routledge, 1985: 195-205, 107-9

23 For a full discussion of how other Articles might be engaged see Hagger L. Some implications of the Human Rights Act 1998 for the medical treatment of children. Medical Law International 2003;6(1):25-51.

24 Douglas and Others v. Hello! Ltd [2001] 2 All ER 289 and Campbell v Mirror Group Newspapers Ltd [2002] EWCA Civ. 1373.

251995 (Cm 5207(1)).

26 www.doh.gov uk/publications, December 2001.

27 See, for example, Royal College of Paediatrics and Child Health. Advocating for Children. London: RCPCH, 2000.

28 The need for such involvement for these purposes receives official recognition in The Special Educational Needs Code of Practice, DfEE (2001), for example.

29 Gersch I. Resolving disagreement in special educational needs: a practical guide to conciliation and mediation. Routledge/Falmer, 2002.

30 Eiser C. Changes in understanding of illness as the child grows. Arch Dis Child 1985;60:489-492; Fielding D, Duff A. Compliance with treatment protocol: interventions for children with chronic illness. Arch Dis Child 1999;80:196200; Alderson P, Montgomery J. What about me? Health Service Journal April 1996:22-4.

31 Chapman M. Constructive evolution: origins and development of Piaget's thought. Cambridge University Press, 1988 (in which he provides a more accurate assessment of Piaget's evolving theories on child development).

32 See, for example, Brook G. Children's competency to consent: a framework for practice. Paediatr Nurs 2000;12(5):31 in which she discusses how such a framework of practice was established at the Liver Unit, Birmingham Children's Hospital NHS Trust.

33 B v. Croydon HA [1995] 1 FCR 332

$34 \mathrm{DoH}$. Recommendation 16, The report of the Victoria Climbié Inquiry. Department of Health, 2003.

35 DoH. Working together to safeguard children: new government proposals for inter-agency co-operation consultation paper. Department of Health, 1998.

36 See comments by Butler Sloss $\sqcup$ in Re G (a minor) [1996] 2 All ER 65 at 68.

37 Caldicott and Data Protection Registrar has produced a checklist for setting up information sharing arrangements reproduced at Appendix 4, Working together document (supra n. 36); see also, Draft guidance on the disclosure of information about sex offenders who may present a risk to children and vulnerable adults. Home Office, July 1999.

38 In A Health Authority $v$ X \& others [2001] 2 FCR 634 the Court of Appeal affirmed that it was lawful to disclose patients' GP records to a health authority to ensure serious allegations were properly investigated.

39 See, for example, W v. Egdell [1990] 1 All ER 835. CA

40 General Medical Council. Confidentiality. London: GMC, 2000. 